\title{
Ulrich Bachmann/Anja Trebes Der Rat der Ratlosigkeit oder: Was der Parlamentarische Rat außer dem Grundgesetz noch hinterlassen hat
}

Es war das Jahr 1948. Die 61 Männer und vier Frauen des Parlamentarischen Rates begannen mit ihren Arbeiten am Grundgesetz. Im Mai 1949 wurde es verabschiedet. Das ist bekannt. Weitgehend unbekannt sind die rund 25000 Seiten Protokolle und Materialien, die die Abgeordneten uns neben den 146 Artikeln hinterlassen haben. Wer will die auch schon lesen? Mitarbeiterinnen und Mitarbeiter der Forschungsstelle für Zeitgeschichte des Verfassungsrechts in Hannover quälen sich durch den auf Schreibmaschine getippten Papierwust, um ihn neu zu ordnen - nach Artikeln sortiert und natürlich auf Diskette. Die so aufbereiteten Materialien werden demnächst in einer mehrbändigen Dokumentation erscheinen.

Was die Abgeordneten alles so von sich gaben, läßt einen nicht nur hier und da schmunzeln, sondern auch gelegentlich zweifeln, ob sich nicht einige mit der Aufgabe überfordert fühlten. Nicht alle waren so offen wie Dr. Becker, der sagte: „Ich gestehe offen, wenn ich gewußt hätte, daß ich hier sechs Monate säße, hätte ich dieses Amt nicht übernommen. « $\mathrm{Zu}$ seiner Ehrenrettung muß man hier allerdings hinzufügen, daß er dies als Vorsitzender des Wahlrechtsausschusses äußerte, eines Ausschusses, bei dem es »wirklich kein Genuß ist, Vorsitzender zu sein ", da "alle drei Stunden etwas anderes beschlossen werden soll«. Aber er tröstete sich selber: »Es gehört nur dazu, daß man frisch und fröhlich an die Dinge herangeht. «

Der Abgeordnete Heiland sprach schon damals die Befürchtung aus: "Ich habe die Sorge, ob man noch mit dem notwendigen Ernst yon uns redet, wenn einmal später unsere Protokolle hier gelesen werden.« Heilands Sorgen war nicht ganz unbegründet. Was zum Beispiel wollen uns die Worte Adenauers sagen: "Der Siebenerausschuß ist tot, es lebe der Siebenerausschuß«? Das wird wohl ein Rätsel bleiben. Nicht ganz so kompliziert waren die bohrenden Fragen Schröters: „Bis wann ist der Parlamentarische Rat gestorben? Wann ist der Parlamentarische Rat tot? « Oder der Satz eines führenden Richters: "Wir brauchen weniger Menschen, ganz abgesehen von den Häusern. « Andererseits gab es auch Erkenntnisse von großer Weisheit: "Die Tatsache, daß zwei Menschen nebeneinander leben, ohne sich zu erschlagen, ist ein Ausdruck des Kompromisses. « Und selbstkritische Reflexion der eigenen Arbeit unter dem modernen Gesichtspunkt des Schutzes der natürlichen Lebensgrundlagen! So besorgte sich Dr. Kleindinst um die Frage des Holzeinschlages, »um die alles kreist. ... Stellen Sie sich vor, was allein die Ausdehnung unserer demokratischen Verfassungen für einen Bedarf an Zeitungspapier bewirkt hat."

Mit der Logik und dem Formulieren hatten einige so ihre Schwierigkeiten. Sätze, die heute im Grundgesetz etwas hölzern wirken, aber dennoch lesbar sind, haben die Abgeordneten einiges Gehirnschmalz gekostet. Heuss gab sich als Verfechter einer klaren Sprache aus. Entschieden wetterte er gegen Partizipialkonstruktionen lateinischer Herkunft im Grundgesetz. »In der ersten Fassung... war das ja nur Partizipialkonstruktion mit einem unmöglichen Satz mit der Gefahr des Stotterns oder Erstickens zwischendrin, wenn man es lesen wollte." Seine Forderung lautete demzufolge: "Die Leute müssen dem Gedanken logisch folgen können, ohne daß sie Atembeschwerden kriegen. "Für eine deutsche Verfassung scheinbar sehr viel ver- 
langt, denn schon in der nächsten Sitzung sah sich Heuss mit Carlo Schmid konfrontiert, der partout den gestelzten Genetiv in Artikel 20 wünschte. Schmid: "Ich verstehe nicht die Kritik an den Genetiven. Ist es nicht schön zu sagen sein Mann großer Kraft ‘ oder seine Frau großer Schönheit ? Man sollte doch den Reichtum unserer deutschen Sprache nicht mit Gewalt an seiner Entfaltung hindern. Der Genetiv hat etwas durchaus Aristokratisches. Man sollte ihm gelegentlich ein Denkmal setzen.« Wo immer dieses Denkmal jetzt steht, in Artikel 20 zumindest nicht.

Eine der schwierigsten Aufgaben bestand darin, sich für ein Wahlsystem zu entscheiden: Sollte es die Mehrheitswahl oder lieber die Verhältniswahl sein? Worin der Unterschied liegt, erklärte der Vorsitzende des Wahlrechtsausschusses, Dr. Becker, in drei Sätzen: »Es wurde einmal gesagt, die ganzen Wahlsysteme unterscheiden sich nur durch die Verlagerung des Kuhhandels: Im Einmannwahlkreis kommt der Kuhhandel vor der Aufstellung, bei der Stichwahl vor dem zweiten Wahlgang; schlössen sich mehrere Parteien zu einer Partei zusammen, dann komme der Kuhhandel in der Fraktion, und bildeten mehrere Parteien die Regierung, dann komme der Kuhhandel in der Koalition. Irgendwo komme er immer. Nur muß man den Kuhhandel vom Kompromiß unterscheiden. Es gibt beides. « Becker hatte gesagt, was es zu dem Thema zu sagen gibt. Alle hatten es verstanden. Theoretische Abhandlungen wurden spätestens an dem Punkt überflüssig.

Das Problem bestand lediglich noch darin, daß das Wahlsystem nicht nur einer parlamentarischen Demokratie gerecht werden mußte, sondern auch für die Bürgerinnen und Bürger draußen im Lande einfach zu verstehen sein sollte. Für sehr intelligent hielt man diese nicht. Man wollte vielmehr »den Staat vor Auswüchsen unbeschreiblicher Dummheit sichern «, wie Rönneburg sich ausdrückte. Sein Kollege Löbe stand ihm da in nichts nach. Als es um die Frage ging, ob man bei einer Wahl seine Stimme mit drei verschiedenen Wertigkeiten auch noch dem gleichen Kandidaten geben könne, meinte Löbe: »Ich möchte vor einer Kumulation mit 1, 2 und 3 warnen. Das ist wirklich kompliziert. Wenn wir im Wahllokal stehen, wissen wir, wie viele Leute unbeholfen hinkommen: Die Alten, die Kranken, die Frauen werden hingeschleppt ... Auf diese Erschwerungen müssen wir also verzichten. «Im übrigen müsse man, so Dr. Greve, »einen gewissen Dusseligkeitskoeffizienten immer einkalkulieren". So sieht das auch sein Kollege Walter: "Schon wenn Sie drei Stimmen geben, ist es kompliziert. Sie wissen so gut wie ich, auf welch tiefern Niveau unser Durchschnittswähler steht. ... Sobald sie etwas schreiben müssen, genieren sie sich im Wahllokal und es gibt Schwierigkeiten. "Andererseits kann man nach Ansicht von Dr. Diederichs »der Inferiorität nicht so weit nachgeben, daß man die Leute nur noch mit einer Hilfe hingehen läßt, die ihnen zeigt, wohin sie ein Kreuz machen sollen.« Mehr als Kreuze sollten sie aber auch nicht machen dürfen, denn Stimmzettel mit Vermerken sind in jedem Fall ungültig. Der Abgeordnete Stock verstand ohnehin nicht, warum jemand auf den Stimmzettel schreibt: "Die Abgeordneten sind alle Lumpen «. Darauf die Erklärung Schröters: »Das kann ich verstehen - natürlich ohne es mir zu eigen zu machen -, er verfolgt damit eine gewisse Absicht.《

Dennoch hat man Wert auf die Stimme des einzelnen gelegt. Harte Sanktionen sollten allen drohen, die nicht wählen gingen. „Der Feigling und Drückeberger muß hart angefaßt werden «, so Heile, der auch schon wußte, wie der Staat den einzelnen zur Wahl scheuchen kann: »Man braucht nur z. B. bestimmen, daß der, der seiner Wahlpflicht nicht nachgekommen ist, höhere, am besten gleich doppelte Einkommensteuer zu zahlen hat. Das wäre ein heilsamer und wirksamer Zwang. "Nun, von dieser Art der Zwänge sind wir noch einmal verschont geblieben. 
Aber nicht nur vom Wahlvolk hatte man keine besonders gute Meinung. Auch den

Männern und Frauen, die sich zur Wahl aufstellen ließen, traute der Rat nicht recht über den Weg. Denn »bei der Prüfung der Wahl haben wir eine Unmenge von Leuten festgestellt, die gleichzeitig Mitglieder zweier Parteien sind «. Woran das lag? Ganz einfach: "Da in Niedersachsen vorgeschrieben war, daß Kandidaten, die auf dem Wahlvorschlag einer Partei kandidieren wollten, dieser Partei angehören mußten, haben sie ihren Eintritt erklärt, um dort aufgestellt zu werden, obwohl sie einer anderen Partei angehörten. «Dr. Diederichs rechtfertigte dieses Gesetz: »Das halten wir für eine Forderung der Ehrlichkeit und Sauberkeit«, und spontan fügte er hinzu: "Wenn ich Zahnpasta verkaufe, und es ist Stiefelwichse in der Tube, so ist das nicht korrekt."

Für sehr kompetent hielt man selbst die Zunft der Juristen nicht, wenngleich hier zuweilen feinsinnig differenziert wurde, wie etwa von Dr. de Chapeaurouge: "Soweit ich sehen kann, sind keine aktiven Richter unter uns, sondern wir sind alles Juristen. « Dr. Wolff, als Präsident des Obersten Gerichtshofes für die britische Zone Sachverständiger des Parlamentarischen Rates, umschrieb das folgendermaßen: "Wir leben nun einmal in einer unvollkommenen Welt und müssen uns damit abfinden, daß ein Urteil falsch ist. « Aber das, so klang durch, ist doch weniger von den damaligen Richtern als wieder vom lästigen Volk zu verantworten: „Der Deutsche hat nun einmal eine große Prozeßstreitsucht«. Welche Berufsauffassung ein Richter hat, darüber gab Dr. Ruscheweyh, ebenfalls Sachverständiger, Auskunft: »... als Richter in der britischen Zone darf ich mich mit Politik nicht beschäftigen. Infolgedessen darf ich auch keine politischen Gedanken haben. «Infolgedessen hat er auch keine, oder wie? Da sich also Juristen nicht mit Politik beschäftigen, wendet sich Ruscheweyh lieber »rein juristischen Fragen « zu: »Nehmen wir an, über einen Grenzgraben zwischen Schleswig-Holstein und Niedersachsen führt eine Brücke, und man streitet sich darüber, ob die 2,50 Mark für ein neues Brett in der Brücke Schleswig-Holstein oder Niedersachsen bezahlen muß.« Darauf Strauß prompt: »Das kann ich sofort durch... Artikel 44 beantworten, wo es heißt: >Das Bundesverfassungsgericht entscheidet ... auch über Streitigkeiten ... zwischen verschiedenen Ländernґ» Ruscheweyh: „Es ist doch gräßlich, die Frage betreffend dieses kleinen Grabens dem Bundesverfassungsgericht zu geben.« Strauß: „Es ist gräßlich. Aber ich muß sagen, es würde gegen die beiden beteiligten Länder sprechen, wenn sie in einem solchen Fall nicht in der Lage wären, sich zu verständigen, und das Bundesverfassungsgericht anrufen müßten. Das wäre entsetzlich. Andererseits gibt es Grenzfälle. Ich meine nicht Fälle der Grenzziehung, sondern äußerste Fälle.« Und sich alle möglichen äußersten Fälle zu erdenken und sie zu regeln, obwohl sie in der Wirklichkeit nicht vorkommen, ist, wenn schon nicht immer Aufgabe, so doch mit Freuden Hobby jedes Juristen. So lange zumindest, bis einer - im Wahlrechtsausschuß war es Schröter - sich gemüßigt fühlte zu fragen: "Ist es denn unsere Aufgabe, auch diese letztmöglichen Fälle in Erwägung zu ziehen? Ich glaube, das ist nicht unsere Aufgabe. "Worauf ihm ein entnervter Vorsitzender beipflichtete: "Ich bin der Letzte, der hier die äußersten theoretischen Dinge hereinbringen will, weil ich auf dem Standpunkt von Schiller stehe: ,Wer allzuviel bedenkt, wird wenig leisten. Wir gehen diesen Weg mit Erfolg seit Wochen.*

Dennoch haben sie bis zum Ende wacker durchgehalten. Selbst den Sitz des Bundestages haben sie bestimmt. Und welche Anstrengung (und Schmiergelder nach dem Menzelschen Motto »Der Geldbeutel ist der wichtigste Körperteil«?) das bedeutete, können wir uns heute lebhaft vorstellen. Da warnte dann auch Schrage den Vorsitzenden des Wahlrechtsausschusses: "Hüten Sie sich davor, in dem Ausschuß die Leitung zu kriegen, in dem der Sitz des Bundestages festgesetzt wird, Bonn oder 
Frankfurt!« Darauf der Vorsitzende: „Ach, das wäre nicht so schlimm!« Und Stock hatte gleich die Lösung parat: »Da muß man vom praktischen Standpunkt ausgehen, von weiter gar nichts. Es handelt sich nur um die Frage: wo ist es möglich, dieses Bundesparlament unterzubringen? «Dr. Greve: »Da wir gute Föderalisten sind, hatte ich gestern Hannover vorgeschlagen!« Stock: »Weil man, wenn man dorthin kommt, nicht einmal als Einzelperson übernachten kann!«

Alle Auseinandersetzungen in den Ausschüssen und im Plenum wären aber wahrscheinlich nur halb so vehement geführt worden ohne den Abgeordneten Renner. $\mathrm{Daß}$ er Mitglied der KPD war, war schon unverzeihlich genug. Aber wie er oftmals analytisch dachte und die Dinge auf den Punkt brachte, ärgerte andere, besonders Helene Wessel, die sich beklagte: »Es scheint mein Schicksal zu sein, daß ich immer hinter dem Abgeordneten Renner sprechen muß. «Als der Parlamentarische Rat von einem Memorandum erst erfuhr, nachdem es der Presse schon längst bekanntgegeben war, erlaubte Renner sich, „diesen hohen Rat hier umzubenennen in Rat der Ratlosigkeit oder in Rat der Menschen, die nicht mehr Rat wissen oder in Haus der Überraschungen. "Wer gegen Renners spitze Zunge nicht ankam, beschloß kurzerhand, ihn zu ignorieren, wie der Abgeordnete Süsterhenn: "Ich habe Ihre Vorlage noch nicht zur Kenntnis genommen, aber ich mißbillige sie von vornherein. "So klangen dann auch die Geburtstagsglückwünsche des Vorsitzenden Schmid, der sich mit Renner im übrigen viele amüsante Wortgefechte lieferte, etwas zu formal: "Ich habe auch heute die Freude, einem Mitglied unseres Ausschusses die Glückwünsche seiner Kollegen aussprechen zu können. Es ist der Kollege Renner, den ich hiermit herzlich zu seinem neuen Lebensjahr beglückwünsche. Möge ihm die Kraft beschieden bleiben, mit der er uns bisher so nützliche Dienste hat leisten können. "Darauf Renner: »Ich darf Ihnen danken, Herr Vorsitzender, nachdem ich bisher von Ihnen in der Hauptsache nur Ordnungsrufe bekommen habe. Deshalb freut mich dieser Glückwunsch ganz besonders. Seien Sie überzeugt, er geht mir so zu Herzen, wie er Ihnen von Herzen kommt."

Wenn die Abgeordneten nicht mit Renner stritten, dann stritten sie über Steuern. Besonders über die Biersteuer. Der Finanzsachverständige Ringelmann wußte zu berichten: „In Bayern spielt das Bier eine ganz andere Rolle als in der überwiegenden Mehrzahl der Länder. Das Bier ist hier ein Volksnahrungsmittel geworden. Bayern war soweit, daß die Bevölkerung an der Preisgestaltung des Biers den regsten Anteil genommen hat. « $\mathrm{Da}$ in Bayern ein Drittel der gesamten Biersteuer aufkommt, wurde dem in einem Artikel Rechnung getragen: »Bei der Aufteilung der Biersteuer sind die besonderen Interessen des Landes Bayern zu berücksichtigen. "Diese Regelung galt allerdings nur bis zum Inkrafttreten des Bundesfinanzausgleichsgesetzes; sie wurde dann überflüssig - und gestrichen.

Aber nicht nur die 6, Abgeordneten machten sich Gedanken über eine zukünftige Verfassung; auch die Bevölkerung schrieb rege Briefe an den Parlamentarischen Rat. So wollte ein Herr Hansen gerne folgende Bestimmung im Grundgesetz wiederfinden: "Wer nicht arbeiten will, bekommt nichts zu essen." Als Erklärung fügt er hinzu: "Ein Naturgesetz Universalgesetz. Zu den politischen und wirtschaftlichen Auswirkungen möchte ich mich erst dann äußern, wenn es gewünscht wird.« Es wurde nicht gewünscht, sein Vorschlag blieb unberücksichtigt. Genauso wie der eines Lübecker Hautarztes, der wollte, daß »jeder Deutsche in verantwortlicher politischer Stellung (Staatspräsident, Ministerpräsident, Minister, General etc.) in Abständen von zwei Jahren ein Zeugnis beizubringen hat, in dem ihm die volle körperliche und geistige Gesundheit bescheinigt wird. «Vielleicht wußten die Abgeordneten schon zu der Zeit, warum sie diesen Vorschlag lieber nicht annehmen sollten. 
Auch zu der Frage des damaligen Frauenüberschusses kamen Anregungen. So die des Kaufmanns Kolb, der die Lösung ganz einfach darin sah, daß ein Mann zwei Frauen heiraten könne. Als Begründung führte er an: ${ }^{x}$ "... jede Frau möchte einen Mann dem Sie gehört, und es ist nachgewiesen und zu vertreten, daß sich zwei Frauen in einen Mann teilen können. Das deutsche Volk in seiner Stellung ist wirtschaftlich jetzt schwach, $u$. in manchem Haushalt und Betrieb wäre eine zweite Frau von Nutzen u. Segen. «Der Kaufmann spricht! Er fuhr fort: »In einem anderen Fall ist eine Frau krank, wo alles Not leidet u. dem Manne bleiben die höchsten Lebensgüter versagt, die Frau kann auch nicht dafür daß sie krank ist. Der Mann kann sich von dieser Frau doch nicht trennen (scheiden lassen) wo sie hilfsbedürftig ist, aber wenn eine zweite vorhanden, hat der Mann eine Frau und die eine Frau eine Pflege u. Unterstützung. « Er endet mit den Worten: »Diese Möglichkeit wenn in der Verfassung verankert u. aufgenommen wird, würde viel Unheil, Ärger, Sorgen und Leid verhüten, die Moral und die Liebe heben.« Was wäre die Welt ohne Männer, die sich so um das Wohl der Frauen sorgen!

Eine Fachlehrerin aus Lebenstedt hätte dem Vorschlag des Herrn Kolb sicher nicht zustimmen wollen. Sie wollte das Problem anders lösen: „Die Reaktion auf den ersten Weltkrieg, der ja ebenfalls einen Frauenüberschuß erzeugte, war eine viel klügere: Es kam das Zeitalter der `Garconne`, des jungenhaften Mädels im Hemdkleidchen - Stoffe waren damals auch knapp - und Bubikopf, das frisch und unbekümmert ihrem Beruf nachging, das sportliebend, wanderfroh, reiselustig und voller Ideale war und sich gar keine Sorgen darum machte, ob es später einmal Kinder haben würde.«

Es sollte noch einige Zeit dauern, bis sich das Frauenbild in der Bundesrepublik änderte. Nicht nur, daß "Frauen im allgemeinen sich politisch nicht interessieren und ihre Stimme nicht den Frauen, sondern den Männern geben«, wie der Abgeordnete Heile versicherte, der nach eigener Bekundung »schon vor vielen Jahrzehnten für die Gleichberechtigung der Frau gekämpft« hat. Es gab von den »Herren Abgeordneten « (wie Adenauer zu sagen pflegte) auch keinen Widerspruch zur Ansicht des Abgeordnete Kaufmann, daß »die Durchschnittsfrau sich aufgrund ihrer Eigenart und ihrer Tätigkeit politisch unmöglich so orientieren kann, wie wir Männer es tun. Sie ist zu ihrer politischen Orientierung auf gelegentliche Unterhaltungen mit der Nachbarin oder dem Manne, wenn er abends müde nach Hause kommt, angewiesen." So ist es kaum verwunderlich, daß der heute eher selbstverständliche Artikel 3 Absatz 2 des Grundgesetzes (»Männer und Frauen sind gleichberechtigt«) erst in der dritten Lesung nach zähem Kampf und Körben voller Protestbriefe verabschiedet wurde. Mann wehrte sich mit Händen und Füßen, obwohl doch alles nur ein Provisorium sein sollte - oder doch nicht? Dachte die Mehrheit wie der Abgeordnete Dr. von Brentano? "... und ich glaube, wir können hier auch etwas aussprechen und sollten es auch aussprechen: die Verfassung, die wir hier beraten, soll ja zwar die gesamtdeutsche Entwicklung nicht präjudizieren, aber wir wollen doch hoffen, daß wir ein Instrument schaffen, das im Grundsatz so ordentlich und gut sein wird, daß es einmal, wenn nicht ohne weiteres als gesamtdeutsche Verfassung übernommen, so doch als Grundlage einer gesamtdeutschen Verfassungsdiskussion betrachtet werden kann; und da müssen wir uns doch klar sein, daß beispielsweise in der russisch besetzten Zone eine Entwicklung eingeleitet ist, die wir dann dort vorfinden werden und für die es gut wäre, wenn in der Verfassung bereits eine Grundlage geschaffen wäre, um dort die geschaffenen Grundlagen zu beseitigen. " Das Protokoll vermerkte in Klammern: Zustimmung und Heiterkeit. Wie wahr.

I Die Orthographie des Orıgınals wurde beibehalten (Red.). 